\title{
Pregnancy-associated breast cancer as a screening and diagnostic challenge: a case report
}

\author{
Anastasia Kalantarova, Nicole Josephine Zembol, Joanna Kufel-Grabowska
}

Department of Electroradiology, Greater Poland Cancer Center, Poznan, Poland

Pregnancy-associated breast cancer (PABC) is the most common malignancy of pregnancy, affecting 1 in 3000 women. Due to the increased size and density of the breast tissue during pregnancy and lactation, diagnosis and treatment are commonly delayed. A 37-year-old woman, gravida 1 para 0 , at the $27^{\text {th }}$ week of gestation presented with two tumors of approximately $2 \mathrm{~cm}$ in the right breast with ipsilateral lymph node involvement on the ultrasonography. HER2-, ER+, PR+, a poorly differentiated ductal carcinoma was identified by the core biopsy and immunohistochemistry. The diagnosis of PABC was made, the tumor's clinical stage was CT2, N1, Mx. She underwent a total mastectomy with axillary node dissection on the right side and was started on adjuvant therapy with paclitaxel. Our report highlights the importance of proper breast oncology surveillance during pregnancy, using safe and inexpensive methods including ultrasonography and biopsy of suspicious masses, to avoid cancer development and progression.

Key words: breast neoplasms, pregnancy, diagnosis

\section{Introduction}

Pregnancy-associated breast cancer (PABC) is a subtype of BC diagnosed in women during pregnancy, first year postpartum, or during the breastfeeding period [1]. Although PABC is thought to have a high mortality rate due to high metastasis rates, potentially related to delays in diagnosis, other factors should be considered. Pregnancy per say does not worsen the prognosis of breast cancer. When matching pregnant and non-pregnant breast cancer patients based on age and tumor advancement, the two populations had similar prognosis given patients were treated with standard BC treatment [2]. With that said, BC patients diagnosed within 2 years postpartum were more likely to present with cancer subtypes associated with poor prognosis (i.e. HER2+, and cancer with basal-like features) compared to both nulliparous controls and patients diagnosed more than 2 years postpartum [3]. Another study reports PABC to have different biological features compared to non-PABC, with poor prognosis reported in $\mathrm{PABC}$ patients with luminal B (HR+ HER2- high Ki-67) and HER2+ cancer subtypes [4].

It has become the most common malignancy of pregnancy, with 1 in 3000 pregnant women affected every year. Of all women diagnosed with breast cancer under 40 years of age, $10 \%$ of women are diagnosed with PABC. Incidence of $P A B C$ is expected to increase over the next years, which may be attributed to delayed conception and family planning, putting women at risk of malignancy due to their increased age. Mortality rates with $\mathrm{PABC}$ are also expected to rise due to the relationship of delayed childbearing. Other important risk factors include no history of breastfeeding, and a family history of BC [5].

The most common presentation of PABC in pregnant women is a palpable lump identified during self-examination,

\section{How to cite:}

Kalantarova A, Zembol NJ, Kufel-Grabowska J. Pregnancy-associated breast cancer as a screening and diagnostic challenge: a case report. NOWOTWORY J Oncol $2021 ; 71: 162-164$.

This article is available in open access under Creative Common Attribution-Non-Commercial-No Derivatives 4.0 International (CC BY-NC-ND 4.0) license, allowing to download articles and share them with others as long as they credit the authors and the publisher, but without permission to change them in any way or use them commercially. 
which is similar to other young women with $B C$ [6]. Unlike BC, the detection of PABC during pregnancy and lactation is possibly confused with normal breast changes, such as an increase in the density and size of the breast parenchyma [7]. Thus, PABC is commonly diagnosed at a more advanced stage and more often with metastasis to the lymph nodes than $B C$. The delay in diagnosis in PABC was reported to be between 1 to 13 months [8]. A clinical breast exam, mammography, breast MRI and ultrasound are all methods available for BC screening [9].

The screening and diagnosis of PABC pose special challenges due to the physical changes in the breast during pregnancy and breastfeeding, and the fetus's risks. As mentioned above, many methods are available for screening, and all vary in their degree of accuracy and safety. The screening method's decision is ultimately at the clinician's discretion and involves combining information about the clinical presentation with patient risk factors. Thus, the lack of a universal screening method for suspected PABC commonly results in a presentation at a more advanced stage and subsequently a poorer prognosis [10]

\section{Case study}

A 37-year-old woman, gravida 1, para 0, presented to an oncology clinic at 27 weeks of gestation. She complained of painless nodules in the right breast detected during self-examination. There was no previous history of nipple discharge or breast disorders and no family history of breast or ovarian cancer. The initial ultrasound investigation was ambiguous. Three months later, a follow up clinical breast examination revealed enlargement of previously suspicious nodules and the appearance of new nodules in the axillary region. An exploratory ultrasonography revealed two spiculated nodules measuring $22 \times 11 \mathrm{~mm}$ and $18 \times 15 \mathrm{~mm}$ in the right breast along with two hypoechoic ipsilateral lymph nodes. There were no nodules in the left breast, aside from a solid cystic focal lesion. A core needle biopsy was performed to confirm the central and upper quadrants of the right breast, and an invasive ductal carcinoma of non-specified type was observed in the central portion of the breast, with an invasion of the nerve trunk. A core biopsy and immunohistochemistry of the lump confirmed HER2-, $\mathrm{ER}+$ and PR+ tumor cells positive for E cadherin.

The tumor's clinical stage was determined to be CT2N1, Mx. Metastatic status to the patient's bones and lungs was not assessed due to the high fetal risk associated with an X-ray and scintigraphy. The clinical presentation of this patient necessitated a right-sided mastectomy and lymphadenectomy. The pre-operative consultation with an obstetrician confirmed that the pregnancy was normal, and there were no alterations in fetal development. The post-surgical pathomorphology confirmed invasive ductal carcinoma diagnosis located in the central portion of the right breast. The pathomorphological report, post-mastectomy, confirmed an invasive ductal carcinoma of non-specified type and the immunohistochemical report verified the HER2-, ER+ and PR+ status of tumor cells.
The results of genetic counseling and testing for BRCA1/2 gene mutations are not yet available. The patient started adjuvant therapy with paclitaxel shortly after the surgery. Recently, our patient gave birth to a healthy baby with no apparent malformations and an APGAR score of 10. A subsequent assessment with a scintigraphy and $\mathrm{X}$-ray post-delivery did not reveal any metastatic lesions.

\section{Discussion}

The attending physician must be highly trained to recognize the wildly under-diagnosed PABC versus the more common hormone-induced breast changes related to pregnancy. A multidisciplinary approach should be used if a woman is diagnosed with PABC to manage her condition while carefully considering the effects on the fetus. This entails psychological counseling due to the intricacy of the issue. Multiple medical specialties should be involved in the treatment plan, including oncology, obstetrics, pediatrics and genetics. A committee should also be available for the patient to discuss any issues relating to psychological impact, religion, or ethics [11]. Having an active group of clinicians and support personnel is a valuable asset for the patient, spouse, family and unborn child.

A clinical breast exam (CBE) is a safe tool for cancer screening during pregnancy and lactation and is routinely performed during a gynecological examination [9]. However, a follow-up assessment with a different radiological technique is often required due to CBE's low sensitivity, especially in high-risk patients [12]. The sensitivity of CBE is likely to increase in breastfeeding women if the examination is performed after pumping or breast-feeding [9]. It is a general recommendation for high-risk women (e.g., older age during pregnancy) to undergo CBE every 6 months during pregnancy and lactation.

A breast MRI is the most accurate BC screening technique with a sensitivity of $71-100 \%$ and a specificity of $89 \%$ [9]. Generally, an MRI is safe to use in pregnant and breastfeeding women since it does not utilize ionizing radiation. The use of MRI in PABC screening is not always advised due to gadolinium's high ability to produce an allergic reaction in the patient. A breast MRI poses little to no safety risk during breastfeeding due to the minimal gadolinium excretion in milk and minimal absorption into the child's digestive system [13]. Generally, a breast MRI is not recommended as a screening tool during pregnancy but is considered safe during breastfeeding, assuming the woman intends to breastfeed more than 6 months postpartum [9].

A mammography is characterized by fairly low sensitivity in pregnant and lactating women [6]. There is limited data available on mammography as a screening tool in pregnancy and breastfeeding [9]. Increased breast density and changes in vascular flow are likely to result in difficulty interpreting the radiographic results [9]. Concerning the developing fetus, mammography poses some risks due to radiation. However, those risks have not been adequately quantified as yet [14]. According to a study conducted in Sweden, a digital mammography can be safely 
used in pregnant women, however only in addition to widely recommended ultrasounds and biopsies [6]. With that said, there are recommendations to evaluate symptomatic women who are younger than 30 years (without regard to pregnancy status) using ultrasonography; the use of mammography is reserved for situations in which ultrasound does not visualize a lesion or the lesion observed is suspicious [15].

A retrospective study conducted in Sweden concluded that PABC patients underwent initial examination with ultrasonography and biopsy more often than mammography [6]. Ultrasound has a diagnostic sensitivity of $100 \%$ with an $86 \%$ specificity when a palpable mass is detected [16]. Although ultrasound has a weak screening sensitivity (29-52\%) in patients not presenting with a palpable mass, many clinicians continue to use it for regular screening in high-risk women during pregnancy and breastfeeding for safety reasons.

A multidisciplinary interplay is especially critical, not only in initial diagnosis, but also in follow up counseling. Some recent studies recommend counseling patients to wait two years after $P A B C$ diagnosis and treatment before planning their next pregnancy due to recurrence risk during pregnancy [1, 17]. Additionally, a study conducted by Clark and Reid of 330 patients concluded that women who waited two years after BC treatment to conceive had a significantly increased five-year survival rate compared with those who waited six months to conceive [18]. While pregnancy appears to confer no increased risk for recurrence of $\mathrm{BC}$, there is no recent data regarding the outcomes of subsequent pregnancy for women with initial PABC. Recurrence of PABC in subsequent pregnancies is an area where future research is essential and can be beneficial in women previously affected with PABC.

\section{Conflict of interest: none declared}

\section{Anastasia Kalantarova \\ Greater Poland Cancer Center \\ Department of Electroradiology \\ ul. 15 Garbary \\ 61-866 Poznań, Poland \\ e-mail:80623@student.ump.edu.pl}

Received: 2 Nov 2020

Accepted: 1 Dec 2020

\section{References}

1. Molckovsky A, Madarnas Y. Breast cancer in pregnancy: a literature review. Breast Cancer Res Treat. 2008; 108(3): 333-338, doi: 10.1007/ s10549-007-9616-6, indexed in Pubmed: 17530426.
2. Beadle $B$, Woodward W, Middleton $L$, et al. The impact of pregnancy on breast cancer outcomes in women $\leq 35$ years. Cancer. 2009; 115(6): 1174-1184, doi: 10.1002/cncr.24165.

3. Sullivan M, Patel A, Wang J, et al. Molecular Subtype Distribution of Pregnancy-Associated Breast Cancer. Am J Clin Pathol. 2013; 140(suppl 1): A091-A091, doi: 10.1093/ajcp/140.suppl1.091.

4. Bae SY, Kim SJ, Lee J, et al. Clinical subtypes and prognosis of pregnancy-associated breast cancer: results from the Korean Breast Cancer Society Registry database. Breast Cancer Res Treat. 2018; 172(1): 113-121, doi: 10.1007/s10549-018-4908-6, indexed in Pubmed: 30088177.

5. Ruiz R, Herrero C, Strasser-Weippl K, et al. Epidemiology and pathophysiology of pregnancy-associated breast cancer: A review. Breast. 2017; 35: 136-141, doi: 10.1016/j.breast.2017.07.008, indexed in Pubmed: 28732325.

6. Johansson ALV, Weibull CE, Fredriksson I, et al. Diagnostic pathways and management in women with pregnancy-associated breast cancer (PABC): no evidence of treatment delays following a first healthcare contact. Breast Cancer Res Treat. 2019; 174(2): 489-503, doi: 10.1007/ s10549-018-05083-x, indexed in Pubmed: 30552644.

7. Taylor D, Lazberger J, Ives A, et al. Reducing delay in the diagnosis of pregnancy-associated breast cancer: how imaging can help us. J Med Imaging Radiat Oncol. 2011; 55(1): 33-42, doi: 10.1111/j.1754-9485.2010.02227.x, indexed in Pubmed: 21382187.

8. Woo JC, Yu T, Hurd TC. Breast cancer in pregnancy: a literature review. Arch Surg. 2003; 138(1): 91-8; discussion 99, doi: 10.1001/archsurg.138.1.91, indexed in Pubmed: 12511159.

9. Carmichael $\mathrm{H}$, Matsen $\mathrm{C}$, Freer $\mathrm{P}$, et al. Breast cancer screening of pregnant and breastfeeding women with BRCA mutations. Breast Cancer Res Treat. 2017; 162(2): 225-230, doi: 10.1007/s10549-017-4122-y, indexed in Pubmed: 28138892.

10. Johansson A, Andersson TL, Hsieh CC, et al. Tumor characteristics and prognosis in women with pregnancy-associated breast cancer. Int J Cancer. 2017; 142(7): 1343-1354, doi: 10.1002/ijc.31174.

11. Amant $F$, Loibl $S$, Neven $P$, et al. Breast cancer in pregnancy. The Lancet. 2012; 379(9815): 570-579, doi: 10.1016/s0140-6736(11)61092-1.

12. Kriege M, Brekelmans C, Boetes C, et al. Efficacy of MRI and Mammography for Breast-Cancer Screening in Women with a Familial or Genetic Predisposition. N Engl J Med. 2004; 351(5): 427-437, doi: 10.1056/ nejmoa031759.

13. Chen MM, Coakley FV, Kaimal A, et al. Guidelines for computed tomography and magnetic resonance imaging use during pregnancy and lactation. Obstet Gynecol. 2008; 112(2 Pt 1): 333-340, doi: 10.1097/ AOG.0b013e318180a505, indexed in Pubmed: 18669732.

14. Butler RS, Chen C, Vashi R, et al. Breast imaging of the pregnant and lactating patient: imaging modalities and pregnancy-associated breast cancer. AJR Am J Roentgenol. 2013; 200(2): 321-328, doi: 10.2214/ ajr.12.9814, indexed in Pubmed: 23345353.

15. Bevers TB, Anderson BO, Bonaccio E, et al. National Comprehensive Cancer Network. NCCN clinical practice guidelines in oncology: breast cancer screening and diagnosis. J Natl Compr Canc Netw. 2009; 7(10): 1060-1096, doi: 10.6004/jnccn.2009.0070, indexed in Pubmed: 19930975.

16. Robbins J, Jeffries $D$, Roubidoux $M$, et al. Accuracy of diagnostic mammography and breast ultrasound during pregnancy and lactation. AJR Am J Roentgenol. 2011; 196(3): 716-722, doi: 10.2214/AJR.09.3662, indexed in Pubmed: 21343518.

17. Azim HA, Santoro L, Pavlidis N, et al. Safety of pregnancy following breast cancer diagnosis: a meta-analysis of 14 studies. Eur J Cancer. 2011; 47(1): 74-83, doi: 10.1016/j.ejca.2010.09.007, indexed in Pubmed: 20943370

18. Clark R, Reid J. Carcinoma of the breast in pregnancy and lactation. Int J Radiat Oncol Biol Phys. 1978; 4(7-8): 693-698, doi: 10.1016/03603016(78)90196-7. 